ISSN 1112-9867

Available online at http://www.jfas.info

\title{
PHYSICO-CHEMICAL CHARACTERISTICS AND QUALITY OF FEED WATERS OF THE EL-OUED CITY STUDY OF CASE (fluorine and Arsenic)
}

\author{
S. E. Laouini*, S. Ladjel and $M^{\text {ed }}$. R. Ouahrani
}

Institut des sciences et technologie, centre universitaire d'El-Oued, Algérie.

Département de génie des procédés, faculté des sciences et sciences de l'ingénieur, Université Kasdi Merbah Ouargla, Algérie.

Received: 19 September 2009 / Accepted: 21 November 2009 / Published online: 31 December 2009

\begin{abstract}
In these days, of numerous country through worlds are confronted to an excess of fluor and arsenic in the wake of drink and preoccupy by in to minimize consequences on the consumers health. The arsenic and the fluor are to scale planetary polluted them of geological origin more frequently met in underground waters. In of numerous countries in expansion, waters so contaminated are often consummated without any previous treatment. Effects inorganic reserved salts in the wake of drink, benefic or luckless acting of their concentration, are known since of numerous years today already more 1,5 billion of persons have not attack to a healthy drinking-water. According to the OMS, these are 15 million to be human who die each year after to drink of the water no potable, or for want of not to have not attack to the drinking-water . Of numerous countries being in possession regions where some waters present amounts in fluor and arsenic superior to recommendations of the OMS $(0,7-1,5 \mathrm{mg} / \mathrm{L}$ for fluor and $10 \mathrm{~L} / \mathrm{g}$ for the arsenic). The fluor behoves against blights and hardens the teeth enamel ;to better concentration it fragilise the bone or induces points of crystallization favoring the appearance of articular distorting arthrosis : this is her fluorose. Effects ought to the arsenic are numerous: lésions of the peel, canker, illnesses cardiovasculair or pulmonarias, hypertension and cancers.
\end{abstract}

Author Correspondence, e-mail: salah_laouini@yahoo.fr

ICID: 1040443 
This work has been realized on the water of five boreholes of the town of El-Oued in collaboration with the laboratory of the waters Algerian (ADE).

The experimentation was aiming to define them plenty of fluor and arsenic exists in five boreholes to study and in fine to estimate the quality consumptive waters.

Key-words: analysis, water qualities, consummation water, fluor, arsenic, Oued Souf

\section{INTRODUCTION}

L'arsenic et le fluor sont à l'échelle planétaire les polluants d'origine géologique les plus fréquemment rencontrés dans les eaux souterraines. Dans de nombreux pays en développement, les eaux ainsi contaminées sont souvent consommées sans aucun traitement préalable [1].

Sur notre planète, la santé de plus d'une centaine de millions de personnes est menacée par l'absorption de doses quotidiennes excessives d'arsenic et de fluor. Ces éléments d'origine géologique se dissolvent dans certaines conditions dans les eaux souterraines à partir substratum constitutif de l'aquifère. Les eaux ainsi polluées sont utilisées dans de nombreux pays en développement pour l'alimentation en eau potable, l'irrigation et la préparation des aliments. De plus, les nappes d'eau souterraines sont de plus en plus sollicitées pour l'adduction d'eau destinée a la consommation, d'une part parce que les ressources en eau potable s'amenuisent de façon générale, d'autre part parce que les eaux souterraines ont la réputation d'être pures car libres de germes pathogènes.

Les nappes d'eau souterraine ne livrent pas toujours une eau absolument potable. En effet, la ou certaines conditions chimiques et géologiques favorisent la libération de substances potentiellement toxiques du substrat minéral dans l'eau souterraine, l'utilisation de cette dernière pour la consommation peut s'avérer très dangereuse pour la santé. Ainsi, en milieu réducteur et pauvre en oxygène, le manganèse et l'arsenic passent sous leurs formes réduites solubles dans l'eau (arsénites pour As). En milieu basique, les anions tels que les fluorures, les arséniates, les vanadates, les séléniates, les borates et les complexes de carbonates d'uranyle se désorbent des surfaces minérales chargées négativement. De plus, les eaux souterraines renfermant peu de calcium dissout peuvent elles aussi présenter des teneurs élevées en anions (fluorures, arséniates, vanadates, etc.).

\section{Synthèse bibliographique}

\section{- Le fluor}


Est l'un des éléments les plus abondants de la croûte terrestre. On le rencontre sous forme de fluorine $\left(\mathrm{CaF}_{2}\right)$, de biotite $\left((\mathrm{Mg}, \mathrm{Fe})_{2} \mathrm{Al}_{2}(\mathrm{~K}, \mathrm{H})\left(\mathrm{SiO}_{4}\right)_{2}\right)$, de cryolithe $\left(\mathrm{Na}_{3}\left(\mathrm{AlF}_{6}\right)\right)$ et de fluoro-apatite $\left(\mathrm{Ca}_{10} \mathrm{~F}_{2}\left(\mathrm{PO}_{4}\right)_{6}\right)$. Ces minéraux étant peu solubles dans l'eau, la concentration des ions fluorure dans les eaux de surface sont généralement faibles. Cependant, les caractéristiques physico-chimiques de certains sels et des nappes d'eau (températures élevées par exemple) au contact de ces roches favorisent la dissolution des minéraux contenant du fluor. La présence d'ions fluorure en excès dans les eaux de boisson est alors à l'origine d'intoxications graves. Comme tout oligoélément, le fluor est nécessaire et bénéfique pour l'organisme humain à de faibles concentrations, mais toxique à plus fortes doses. En effet, à partir de $0,5 \mathrm{mg} / \mathrm{L}$ en ions fluorure, une eau joue un rôle prophylactique, mais dès $0,8 \mathrm{mg} / \mathrm{L}$, le risque de fluorose débute et devient fort au dessus de $1,5 \mathrm{mg} / \mathrm{L}$. La norme admise varie dans un domaine de concentration de 0,7 à $1,5 \mathrm{mg} / \mathrm{L}$ pour des températures de 12 à $25^{\circ} \mathrm{C}$ [1]. Les premières atteintes sont d'ordre esthétique avec des taches caractéristiques sur les dents (fluorose dentaire). Au Sénégal, comme dans de nombreux autres pays du monde, les études montrent qu'à partir de $2 \mathrm{mg} / \mathrm{L}$, tous les enfants sont atteints et que $60 \%$ d'entre eux ont des fluoroses graves.

Pour des teneurs voisines de $4 \mathrm{mg} / \mathrm{L}$. Des atteintes plus graves encore concernant les os et les articulations (fluoroses osseuses) sont observés lorsque l'eau contient plus de $7 \mathrm{mg} / \mathrm{L}$.

\section{- Chimie du fluor dans les eaux souterraines}

Les eaux souterraines se chargent en fluor après lessivage de roches phosphatées probablement par dissolution des apatites fluorées dont la solubilité augmente avec la température des nappes considérées (à $\mathrm{T}>35^{\circ} \mathrm{C}$ ). Le niveau de saturation des eaux dépend principalement du déplacement de l'équilibre (1) de formation de la fluorine

$$
\left(\mathrm{CaF}_{2}\right): \mathrm{Ca}_{2}^{+}+2 \mathrm{~F}^{-} \longrightarrow \mathrm{CaF}_{2}
$$

La teneur en calcium, l'équilibre des eaux en calcite $\left(\mathrm{CaCO}_{3}\right)$, gypse $\left(\mathrm{CaSO}_{4}, 2 \mathrm{H}_{2} \mathrm{O}\right)$, et la, formation de complexes calciques $\left(\mathrm{CaSO}_{4}, \mathrm{CaHCO}_{3}+, \mathrm{CaCO}_{3}\right)$ sont des facteurs importants pour le déplacement de cet équilibre. Les eaux des zones de sud Algérie peuvent être soit sous-saturées, soit sursaturées en fluorine [4]. Pour les eaux soussaturées en fluorine, c'est le rôle de l'écoulement dans le réservoir qui est prépondérant. En effet, un forage peut modifier la nature des écoulements et la vitesse d'échange des 
sels minéraux entre le réservoir et la nappe. Pour les eaux sursaturées, l'équilibre de formation de la fluorine contrôle la concentration en ions fluorure.

\section{- l'Arsenic}

L'arsenic est un élément naturel très répandu dans la croûte terrestre. On le trouve fréquemment à l'état naturel dans les eaux souterraines, par l'érosion et le vieillissement climatique des sols, des minéraux et des minerais. Dans les secteurs commercial et industriel, les composés de l'arsenic entrent dans la fabrication d'une multitude de produits et peuvent contaminer les sources d'eau potable de façon directe par le biais des effluents industriels, et de façon indirecte par des dépôts atmosphériques. La présence d'arsenic dans les eaux souterraines est liée aux caractéristiques géologiques et géochimiques [7]. L'arsenic est présent dans les roches éruptives et métamorphiques. Il est ensuite réparti lors de l'altération des roches [8]. Il est le vingtième constituant en terme d'abondance dans la croûte terrestre et se trouve souvent associé aux minéraux sulfurés : arsénopyrite (FeAsS), réalgar $\left(\mathrm{As}_{2} \mathrm{~S} 2\right)$ et l'orpinet $\left(\mathrm{As}_{2} \mathrm{~S}_{3}\right)$ [9]. L'arsenic a une forte affinité pour les hydroxydes de fer. De fortes teneurs en arsenic peuvent être associées à la dissolution des hydroxydes de fer contenant de l'arsenic [7]. $90 \%$ de l'arsenic est sous forme inorganique [10]. Les deux formes principales sont l'arséniate, As (V) présent sous forme $\mathrm{H}_{2} \mathrm{AsO}_{4}$.

Où $\mathrm{HAsO}_{3}$ et l'arsénite, As (III), présents sous forme $\mathrm{H}_{3} \mathrm{AsO}_{3}$. La forme non chargée de l'arsenic, As (III) est plus toxique et plus mobile.

Les effets dus à l'arsenic sont nombreux: lésions de la peau, gangrène, maladies cardiovasculaires ou pulmonaires, hypertension et cancers. De hautes concentrations peuvent provoquer l'arsenicose [12]. La norme d'OMS est de 10 microgramme par litre ( $\mathrm{g} / \mathrm{l}$ ) pour la teneur dans l'eau potable [13]. Il s'agit aussi de la norme retenue par l'Agence Américaine de Protection de l'environnement (USEPA). La teneur limite en arsenic dans de l'eau brute destinée après traitement à la consommation humaine est fixée à $100 \mathrm{~g} / \mathrm{l}\left(\right.$ Décret $\mathrm{n}^{\circ}$ 2001-1220, 2001). Entre 10 et $13 \mathrm{~g} / \mathrm{l}$ des dérogations sont possibles mais avec une durée limitée et des restrictions d'utilisation (Direction Générale de la Santé, 2005).

\section{MATERIELS ET METHODES}

Les prélèvements de l'eau ont été effectués au cours du mois de mai de l'année 2009. Pendant cette période, les consommations de l'eau est très élevées et le début est 
maximum. Les eaux ont été prélevées directement si le pompage est en cours; sinon après un certain temps depuis le démarrage de la pompe a partir des réservoirs de stockage. Dans les deux cas, on s'est assuré de la stabilisation de la conductivité de l'eau avant chaque échantillonnage. Les échantillons sont prélevés dans des bouteilles en polyéthylène, préalablement lavées avec une solution alcalin et rincées plusieurs fois avec l'eau distillée. Les échantillons ont été conservés à une température de $4^{\circ} \mathrm{C}$. Les mesures sont effectuées au laboratoire de l'algérienne des eaux (unité d'Oued Souf) par méthode spectrophotométrie (spectrophotomètre DR/2500) pour le fluor et spectrométrie d'absorption atomique pour l'arsenic [12].

\section{Dosage du fluore}

Le dosage du fluor est réalisé par méthode spectrophotométrie, on utilise l'appareillage DR/2500. En introduit les échantillons dans l'appareil et en sélectionne la longueur d'onde et le programme Hach et l'élément fluor, le programme affiche la concentration du fluor de l'échantillon

Pour éviter les contaminations par des produits fluorés, utiliser de préférence pour les prélèvements des flacons neufs en polyéthylène.

\section{EXPRESSION DES RESULTATS}

Les résultats sont exprimés en microgrammes d'arsenic par litre.

La limite de détection est de $0,1 \mathrm{~g} / \mathrm{L}$.
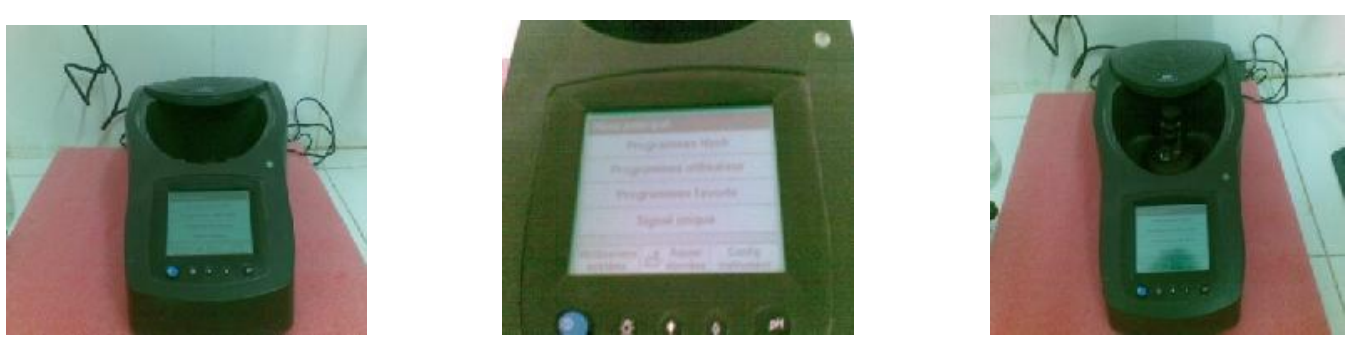

Fig.1. Appareillage utilisé DR/2500

\section{Dosage de l'Arsenic}

On considère que l'absorption atomique après formation d'hydrure gazeux est la méthode la plus appropriée pour doser l'arsenic dans l'eau [3], la limite de détection étant d'environ $1 \mathrm{mg} / \mathrm{L}$. 
Le dosage de l'arsenic demande que tous les composés de l'arsenic soient à la valence III. La totalité de l'arsenic est donc d'abord oxydé à l'état V. L'arsenic (V) est alors réduit quantitativement en arsenic (III) par l'iodure de potassium. Puis l'arsenic (III) est réduit à l'état d'hydrure $\left(\mathrm{H}_{3} \mathrm{As}\right)$ par le borohydrure de sodium. L'hydrure libéré dans le réacteur est entraîné dans un tube en silice chauffé.

\section{MODE OPERATOIRE}

Introduire dans le générateur d'hydrure $20 \mathrm{ml}$ d'échantillon, $1 \mathrm{ml}$ de solution d'iodure de potassium, $5 \mathrm{ml}$ d'acide chlorhydrique et un barreau aimanté recouvert de téflon. laisser stabiliser $40 \mathrm{~s}$ sous courant d'azote ou d'argon puis agiter $20 \mathrm{~s}$ en plaçant le flacon sur agitateur magnétique. Dans cette première phase, l'arsenic (V) est réduit en arsenic (III). Maintenir l'agitation et introduire une pastille de borohydrure de sodium. Mettre le générateur en communication avec le spectrophotomètre d'absorption atomique. Entraîner l'hydrure d'arsenic au moyen du gaz vecteur à un débit de 1 à 3 L/ min dans le tube en silice chauffé dans le brûleur air acétylène ou électriquement. Travailler en correction de bruit de fond, intégrer pendant 45 s. Effectuer les lectures à la longueur d'onde de 193,7 nm.

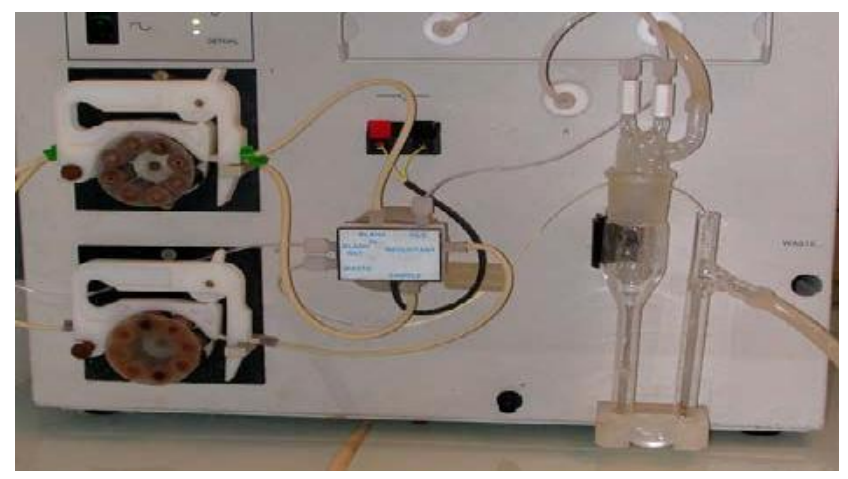

Fig.2. La spectrométrie d'absorption atomique avec génération d'hydrure

\section{EXPRESSION DES RESULTATS}

Les résultats sont exprimés en milligrammes du fluor par litre.

La limite de détection est de $0,01 \mathrm{mg} / \mathrm{L}$.

\section{Résultats d'analyse}

Les résultats d'analyses sont enregistrés dans le tableau suivant: 


\begin{tabular}{|c|c|c|c|c|c|c|}
\hline communes & $\begin{array}{l}\text { Volume } \\
\text { desservit } \\
\left(\mathrm{m}^{3} / \mathrm{j}\right)\end{array}$ & $\begin{array}{l}\text { Nombre } \\
\text { d'abonnés }\end{array}$ & $\begin{array}{l}\text { Source } \\
\text { d'alimentation }\end{array}$ & $\begin{array}{l}\text { Point de } \\
\text { prélèvement }\end{array}$ & $\begin{array}{l}\text { Fluore } \\
(\mathrm{mg} / \mathrm{l})\end{array}$ & $\begin{array}{l}\text { Arsenic } \\
(\mathrm{g} / \mathrm{l})\end{array}$ \\
\hline \multirow{5}{*}{ El-Oued } & 51,840 & \multirow{5}{*}{19181} & \multirow{5}{*}{ forages } & $\begin{array}{l}\text { Forage pontier } \\
\text { Sidi Mastour }\end{array}$ & 2.88 & 3.61 \\
\hline & 20,736 & & & $\begin{array}{l}\text { Forage pontier } \\
\text { Chouhada }\end{array}$ & 2.65 & 2.21 \\
\hline & 51,816 & & & $\begin{array}{l}\text { Forage pontier } \\
\text { Chott }\end{array}$ & 3.54 & 2.35 \\
\hline & 12,960 & & & $\begin{array}{l}\text { Forage pontier } \\
\text { Naoura }\end{array}$ & 3.12 & 2.41 \\
\hline & 51,810 & & & $\begin{array}{l}\text { Forage pontier } \\
08 \text { Mai }\end{array}$ & 2.92 & 2.95 \\
\hline
\end{tabular}

\section{DISCUSSION DES RESULTATS}

D'après les résultats obtenus, on remarque la valeur élevée du fluor dans tout les échantillons, on enregistré la valeur maximum dans Forage pontier Chott avec la valeur de $3.54 \mathrm{mg} / \mathrm{l}$. Toutes les valeurs dépassent les normes d'OMS et l'Agence Américaine de Protection de l'environnement (USEPA). Cettes valeurs présentent des effets indésirables sur la santé des consommateurs comme indique ci-dessus.

La géologie joue également un rôle prédominant dans le phénomène de contamination des eaux souterraines par le fluor [3]. Les roches magmatiques, notamment, peuvent renfermer de fortes concentrations de fluorures. D'autre part, la possibilité de libération de calcium à partir du substrat géologique est un critère important étant donné sa capacité de coprécipitation avec le fluor sous forme de fluorure de calcium. En plus de cela, les conditions climatiques sont à prendre en considération puisque par exemple un climat sec et aride favorise l'accumulation des sels (notamment de fluor) dans les eaux souterraines les plus proches de la surface. Enfin, le $\mathrm{pH}$ du sol est un paramètre important puisque les fluorures sont libérés sous forme anionique en milieu basique [13].

Pou l'arsenic, les valeurs enregistrées (entre 2,21 et 3,61 g/l) sont dessous des normes de OMS et CEE et les valeurs de celui-ci ne présente aucune danger sur la santé des consommateurs. 


\section{CONCLUSION}

Dans ce travail, on prend comme cas une étude physico-chimique de concentration du fluor et arsenic dans les eaux d'alimentation de la ville d'El-Oued. D'après l'analyse, on enregistre la valeur élevée du fluor, cette valeur élevée exige un traitement spécifique pour réduire la concentration du fluor. Plusieurs méthodes utilisées pour diminuer la concentration du fluor dans les eaux potable, parmi les principales méthodes, précipitation, adsorption, échange d'ionique et techniques membranes.

Dans ce stade on cherche sue la méthode plus approprier pour les caractéristiques physico-chimiques des eaux d'alimentation et la méthode moins couteux.

\section{RÉFÉRENCES}

[1] Amini M., Abbaspour K.C., Berg M., Winkel L., Hug S.J., Hoehn E., Yang H., Johnson C.A. Statistical modeling of global geogenic. arsenic contamination in groundwater. Environmental Science and Technology. 2008, 42, 3669 -3675.

[2] Winkel L., Berg M., Amini M., Hug S. J., Johnson C. A. Predicting groundwater arsenic contamination in Southeast Asia from surface parameters. Nature Geoscience. 2008, 1, 536 -542. doi :10.1038/ngeo254.

[3] Amini M., Mueller K., Abbaspour K.C., Rosenberg T., Afyuni M., M ller K.N., Sarr M., Johnson C.A. Statistical modeling of global geogenic fluoride contamination in groundwaters. Environmental Science and Technology. 2008, 42, 3662-3668. doi :10.1021/es071958y

[4] Hug S.J., Leupin O.X., Berg M. Bangladesh and Vietnam : Different groundwater compositions require different approaches for arsenic mitigation. Environmental Science and Technology. 2008, 42. doi :10.1021/es7028284

[5] Müller K., Kage F., Wanja E., Mattle M., Osterwalder L., Zurbrügg C., Johnson C.A. Improving fluoride remocal efficiency. Sandec News. 2008, 9, 6.

[6] Parthasarathy N., Buffle J., Haerdi W. Combined use of calcium salts and polymeric aluminium hydroxide for defluoridation of waste waters. Water Res. 1986, 20(4), 443.

[7] Yang C.L., Dluhy R. Electrochemical generation of aluminium sorbent for fluoride adsorption.J. of Hazardous Materials. 2002, 94(3), 239.

[8] Srimulari M., Pragathi A., Karthikeyan J. A study on removal of fluorides from drinking water by adsorption onto low-cost materials. Environ. Pollut. 1998, 99, 285. 
[9] Wang W., Li R., Tan J., Luo K., Yang L., Li H., Li Y. Adsorption and leaching of fluoride in soils of China. Fluoride. 2002, 35(2), 122.

[10] Agarval M., Rai K., Shrivastav R., Dass S. Defluoridation of water using amended clay. J. of Cleaner Produc. 2003, 11, 439.

[11] Garmes H., Persin F., Sandeaux J., Pourcelli G., Mountadar M. Defluoridation of groundwater by a hybrid process combining adsorption and Donnan dialysis. Desalination. 2002, 145, 287.

[12] Benalaya A, Chkirbene A, Jallali S, Harbaoui K et Tarhouni J.(2009). Evaluation de la qualité des eaus de la nappe coté orientale du Cap-bon en tunisie, 113p .

[13] Lhassani A., Rumeau M., Benjelloun D., Pontié M. Selective demineralisation of water by nanofiltration application to the defluoridation of brackish water. Water Res. $2001,35,3260$. 


\section{CARACTERISTIQUES PHYSICO-CHIMIQUES ET QUALITE DES EAUX D'ALIMENTATION DE LA VILLE D'EL-OUED TUDE DE CAS}

(Fluor et Arsenic)

\section{RESUME}

De nos jours, de nombreux pays à travers le monde sont confrontés à un excès de fluor et arsenic dans les eaux de boisson et se préoccupent d'en minimiser les conséquences sur la santé des consommateurs. L'arsenic et le fluor sont à l'échelle planétaire les polluants d'origine géologique les plus fréquemment rencontrés dans les eaux souterraines. Dans de nombreux pays en développement, les eaux ainsi contaminées sont souvent consommées sans aucun traitement préalable. Les effets des sels minéraux contenus dans les eaux de boisson, bénéfiques ou néfastes en fonction de leur concentration, sont connus depuis de nombreuses années Aujourd'hui déjà plus de 1,5 milliards de personnes n'ont pas accès à une eau potable saine [1]. Selon l'OMS, ce sont 15 millions d'être humains qui meurent chaque année après avoir bu de l'eau non potable, ou faute de n'avoir pas accès à l'eau potable [2] De nombreux pays possédant des régions où certaines eaux présentent des teneurs en fluor et arsenic supérieures aux recommandations de l'OMS (0,7-1,5 mg/L pour fluor et $10 \mathrm{~g} / \mathrm{L}$ pour l'arsenic [3]). Le fluor est utile contre les caries et durcit l'émail des dents ; à plus forte concentration il fragilise les os ou provoque des points de cristallisation favorisant l'apparition d'arthroses articulaires déformantes : c'est la fluorose [4]. Les effets dus à l'arsenic sont nombreux: lésions de la peau, gangrène, maladies cardiovasculaires ou pulmonaires, hypertension et cancers. Ce travail a été réalisé sur l'eau de cinq forages du la ville d'Eloued en collaboration avec le laboratoire de l'Algérienne des eaux (ADE). L'expérimentation visait à définir les quantités de fluor et arsenic existe dans les cinq forages étudié et en fin estimé la qualité des eaux de consommation.

Pour les cinq forages, la concentration du fluore est supérieur les normes fixées par OMS et CEE, les valeurs enregistrées situer entre 2,65 et 3,12 mg/l.

Mots-clés: Analyse, Qualité des eaux, eaux d'alimentation, analyse, fluor, arsenic, Oued Souf

\section{How to cite this article}

Laouini S E, Ladjel S and Ouahrani $\mathrm{M}^{\mathrm{ed}} \mathrm{R}$. Physico-chemical characteristics and quality of feed waters of the El-Oued city study of case (fluorine and arsenic). J Fundam Appl Sci. 2009, 1(2), 13-22. 